\title{
CONTINUOUS CHANGE DETECTION OF URBAN LAKES IN WUHAN, CHINA USING MULTI-TEMPORAL REMOTE SENSING IMAGES
}

\author{
Wenyuan Zhang ${ }^{1}$, Xiaohan Kong ${ }^{1, *}$, Guoxin Tan ${ }^{1}$, Songyin Zheng ${ }^{1}$ \\ ${ }^{1}$ National Research Center of Cultural Industries, Central China Normal University, Luoyu Road, Wuhan, China - \\ zhangwy@mail.ccnu.edu.cn, k13296632062@163.com,gxtan@mail.ccnu.edu.cn, zhengsongyin@126.com
}

Commission III, WG III/7

KEY WORDS: Change Detection, Urban Lakes, Multi-temporal Remote Sensing Images, Modified NDWI, Wuhan City

\begin{abstract}
:
Urban lakes are important natural, scenic and pattern attractions of the city, and they are potential development resources as well. However, lots of urban lakes in China have been shrunk significantly or disappeared due to rapid urbanization. In this study, four Landsat images were used to perform a case study for lake change detection in downtown Wuhan, China, which were acquired on 1991, 2002, 2011 and 2017, respectively. Modified NDWI (MNDWI) was adopted to extract water bodies of urban areas from all these images, and OTSU was used to optimize the threshold selection. Furthermore, the variation of lake shrinkage was analysed in detail according to SVM classification and post-classification comparison, and the coverage of urban lakes in central area of Wuhan has decreased by $47.37 \mathrm{~km}^{2}$ between 1991 and 2017. The experimental results revealed that there were significant changes in the surface area of urban lakes over the 27 years, and it also indicated that rapid urbanization has a strong impact on the losses of urban water resources.
\end{abstract}

\section{INTRODUCTION}

Urban lakes are important freshwater resources available for ecosystems, they provide precious water to residents, fish and waterfowls, as well as regulate the urban environment, i.e., humidity, temperature, flood storage (J. Zhu, Zhang, and Tong 2015; Snehal and Unnati 2012; W. Zhu, Jia, and Lv 2014; Taravat et al. 2016). However, with human activity increasing and rapid urbanization, many urban lakes in China have been shrunk significantly or disappeared in recent years. Thus there is an increasing necessity to understand the dynamical relationship between lake shrinking and urbanization, not only temporally but also spatially for the improvement of urban environments.

Remote sensing, as an advanced technology of Earth observation and an important tool for providing spatially consistent image information, has been widely used in various applications, such as land use or land cover change (Z. Zhu and Woodcock 2014; Demir, Bovolo, and Bruzzone 2013), urban sprawl (Bagan and Yamagata 2012), and hydrology (Dronova, Gong, and Wang 2011; W. Zhu, Jia, and Lv 2014; Rokni et al. 2014). In particular, since the Landsat archive was open to public by the U.S. Geological Survey (USGS) in 2008 (Woodcock 2008), Many studies have focused on water change detection, lake monitoring and lakefront land use classification using multi-temporal Landsat images (Rokni et al. 2014; Taravat et al. 2016; Z. Zhu and Woodcock 2014). For example, Michishita et al. (2012) examined the two decades of urbanization in the Poyang Lake area in China, using a timeseries Landsat-5 TM dataset, and performed a quantification and visualization of the changes in time-series urban land cover fractions through spectral unmixing. Song et al. (2013) estimated the water storage changes in the lakes of the Tibetan Palteau by combining time-series water level and area data derived from optical satellite images over a long time scale, and analysed the changes therein. Zhu et al. (2014) monitored the fluctuation of Qinghai Lake by estimating the variations of water volume based on MODIS (Moderate Resolution Imaging Spectroradiometer) and Landsat TM/ETM+ images from 1999 to 2009. Zhu et al. (2015) quantitatively analysed the impacts of lakefront land use changes on lake area in Wuhan, based on two Landsat TM/ETM+ images taken in 1991 and 2005.

However, only two dates of satellite images were used in most change detection algorithms (J. Zhu, Zhang, and Tong 2015), qualitative analysis of the temporal effects of phenomenon are limited. Moreover, a unified category or single lake were often chosen to monitor in lots of publications (Zheng et al. 2015; W. Zhu, Jia, and Lv 2014). In this paper, we have performed a case study for urban lake change detection in Wuhan, China, using multi-temporal Landsat images. The aim of this study is quantitatively change analysis of urban lakes shrinking and impact of the factors over the past few decades.

This paper starts with a description of our study area, and collected remote sensing datasets, and data pre-processing in Section 2. Section 3 describes our approaches to extract the surface water extent of urban lakes, as well as the land cover classification in the study area by using multi-temporal images. A detail change analysis and the impact of factors about lake shrinking based on experimental results are discussed in Section 4. Section 5 summarizes and concludes the results of this study. 


\section{STUDY AREA AND DATA}

\subsection{Study Area}

Wuhan is located at $113^{\circ} 41^{\prime} \mathrm{E}$ to $115^{\circ} 05^{\prime} \mathrm{E}$ and $29^{\circ} 58^{\prime} \mathrm{N}$ to $31^{\circ} 22^{\prime}$ N. As the capital of Hubei Province, Wuhan is the only sub-provincial city in Central China with an area of 8,494 square kilometres. The world's 3rd longest river-the Yangtze River and its greatest branch, the Hanshui River flow across the city and divide it into three parts, namely Wuchang, Hankou and Hanyang. (Figure 1).

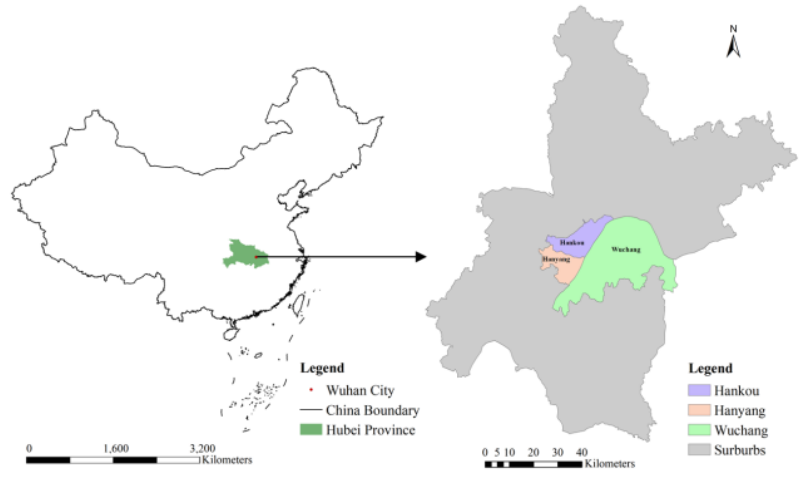

Figure 1. Location and administrative division of Wuhan City.

Wuhan is famous for its lake resources. There are more than 166 lakes now, so it is well known as the City of Hundreds of Lakes. Since the 1990s, Wuhan has witness the rapid urban expansion. During the urbanization process, most urban areas are developed and expanded through the alteration of other land types including lakes, vegetation, and agricultural fields. A statistic report on the lakes in Wuhan suggests that the count of urban lakes has been decreased from 127 to 38,89 lakes have vanished completely during the past 60 years (Wang 2013).

The downtown Wuhan covering Wuchang, Hankou and Hanyang was selected as study area, and its total area was 962.8227 square kilometers. Nowadays, there are only 26 major lakes lie in this region, including the famous East Lake.

\subsection{Data}

In order to interpret the lake surface extent and land-cover change for this study area, four multi-spectral Landsat images covering Wuhan in different periods were collected from USGS website (http://earthexplorer.usgs.gov/). These images were acquired on four separate years from 1991 to 2017 (path/row 123/39), and all of them were cloud free. The characteristics of these images are provided in Table 1. The first two images of the Landsat- 5 satellite was captured by Thematic Mapper (TM) sensor with seven bands, and the third image captured by Landsat-7 Enhanced Thematic Mapper Plus (ETM+) sensor included eight bands. The spatial resolution of TM and ETM+ data was $30 \mathrm{~m}$ for bands $1-5$ and 7 . While TM band 6 and ETM+ band 6 (thermal infrared) were acquired at $120 \mathrm{~m}$ resolution, and ETM+ band 8 (panchromatic) was $15 \mathrm{~m}$ resolution. The last image was from Landsat- 8 Operational Land Imager (OLI) sensor with nine bands, the spatial resolution is $30 \mathrm{~m}$, while excluding one panchromatic band $(15 \mathrm{~m})$. All images had been geo-referenced and projected to the same Universal Transverse Mercator (UTM) coordinate system (UTM zone 49 N, WGS-84 geodetic datum).
Wuhan has a humid subtropical monsoon climate with abundant rainfall, and the annual rainfall is concentrated from late June to early August. Hence, the selected remotely sensed images were acquired after the rainfall and on a similar climate condition.

\begin{tabular}{|c|c|l|c|}
\hline Acquisition Date & Satellite & Sensor & Band Count \\
\hline $1991 / 10 / 23$ & Landsat-5 & TM & 7 \\
\hline $2002 / 09 / 03$ & Landsat-5 & TM & 7 \\
\hline $2011 / 11 / 23$ & Landsat-7 & ETM+ & 8 \\
\hline $2017 / 10 / 30$ & Landsat-8 & OLI & 9 \\
\hline
\end{tabular}

Table 1. Characteristics of data sources

\subsection{Data Pre-processing}

A series of pre-processing procedures were performed on the acquired data. Firstly, a local linear histogram matching technique was used to fill the gaps in the ETM+ image, due to the failure of Landsat-7 scan-line corrector since May 31, 2003. The processed image was spatially continuous without any obvious striping patterns. Secondly, polynomial correction method was applied to reduce or eliminate the geometric distortion of all images. Thirdly, radiation calibration and atmospheric correction based on FLAASH model were performed to all images. Fourth, taking the last image as a reference, a histogram matching method was used to adjust the hue and contrast of the rest images. Finally, all the corrected images were then cropped to cover the whole downtown area of Wuhan by using the same area of interest (AOI), and the image size was $1662 \times 1214$ pixels.

All the aforementioned processing were performed using ENVI (The Environment for Visualizing Images) 5.1 software package. Figure 2 shows an example of these image with specific band combination (near infrared band, red band and green band from each Landsat image).

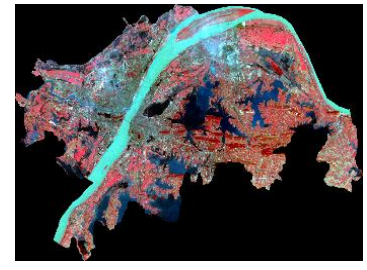

(a)

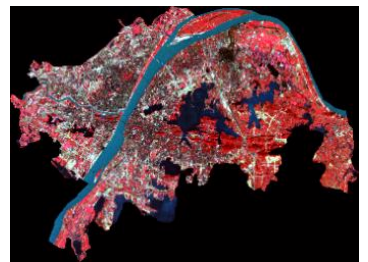

(c)

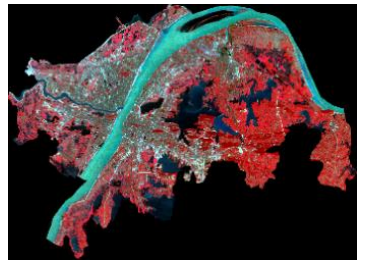

(b)

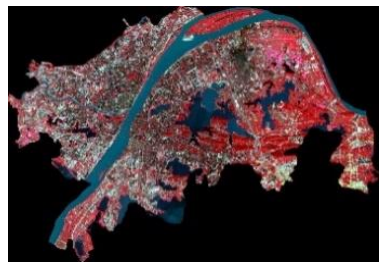

(d)
Figure 2. Multi-temporal Landsat images for the study area: (a) 1991, (b) 2002, (c) 2011, and (d) 2017.

\section{METHODOLOGY}

\subsection{Workflow}

The methodology of this investigation is represented in Figure 3. 


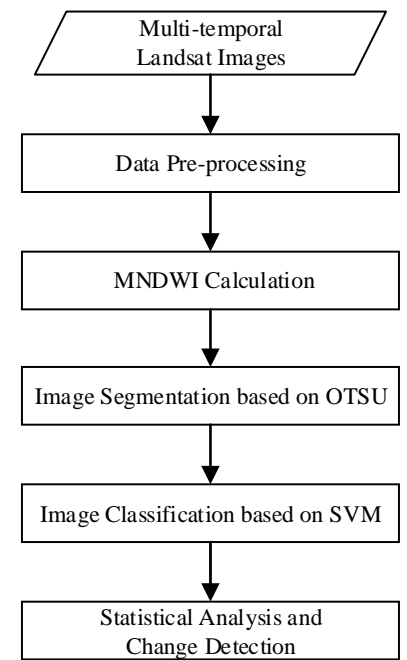

Figure 3. Schematic representation of the methodology

The workflow includes four major stages: MNDWI calculation, image segmentation, image classification, and change detection. First, the MNDWI index was calculated to obtain a gray-scale image for each dataset. Then, OTSU method was used to segment the MNDWI image, and the water bodies could be extracted from the segmented image. Third, classification algorithm such as SVM was adopted to classify the gray-scale image. Finally, a detailed change analysis was performed based on post-classification.

\subsection{Water Surface Extraction}

In recent years, various algorithms have been proposed to extract water information from remote sensing images from different sensors. As we known, the normalized difference water index (NDWI) proposed by McFeeters (McFeeters 1996) is one of the most efficient approaches for water extraction from multiband images, which is calculated as follows:

$$
N D W I=\frac{G R E E N-N I R}{G R E E N+N I R}
$$

Where GREEN is a green band such as TM band 2, and NIR is a near infrared band such as TM band 4 .

However, the extracted water information using NDWI is often mixed with built-up land noise in urban districts, because many built-up land features in Landsat TM/ETM+ images also have positive values in the NDWI image. To overcome the limitation of NDWI, a modified NDWI (MNDWI) was thus introduced by (Xu 2006), which is more suitable for improving open water features extraction from remote sensing images where water regions surrounded by lots of built-up areas. The MNDWI is expressed as follows:

$$
M N D W I=\frac{G R E E N-M I R}{G R E E N+M I R}
$$

Where MIR is a middle infrared band such as TM band 5 .

For Landsat TM/ETM+ image, MNDWI can be formed as:

$$
M N D W I=\frac{B 2-B 5}{B 2+B 5}
$$

Where $\boldsymbol{B} 2$ and $\boldsymbol{B 5}$ are the pixel values of the second and fifth bands in the Landsat images, respectively.

As shown in Equation (1) and (2), NIR in NDWI is replaced by $M I R$ because the water body has lower reflectivity in the middle infrared band than construction. Thus, MNDWI could effectively suppress and even remove built-up features as well as vegetation and soil features ( $\mathrm{Li}$ et al. 2013).

\subsection{Image Segmentation}

Threshold selection is a key step for in defining MNDWI. Because of their high absorption in the MIR band and high reflectance in the Green band, water features usually have positive MNDWI values, while the MNDWI values of nonwater features are usually negative. Thus, the threshold value for Xu's MNDWI was set to zero. However, threshold adjustment in individual scenes may achieve a more accurate delineation of water bodies (Ji, Zhang, and Wylie 2009). Therefore, dynamic threshold values are needed when different regions of remote sensing images are employed to extract water body information. In this study, the maximum between-class variance method (the OTSU method) was adopted to each individual image (Otsu 1979), so as to identify the optimal threshold values for separating water bodies from the background features.

According to the Equation (2), the MNDWI pixels range from $[-1,1]$. Since the OTSU method will lose some of the overall information for two groups of objects in the case of the unidentified grayscale, an enhanced OTSU method using grayscale stretching was adopted to solve this problem. A grayscale stretching strategy was applied to increase the gray level, so as to enhance the gray differences between the first two groups of objects. The stretching method in this study was defined as:

$$
y=\frac{x-x_{\min }}{x_{\max }-x_{\min }} \times 255
$$

After stretching, the MNDWI pixel values range from [0, 255].

\section{EXPERIMENT AND ANALYSIS}

\subsection{Lake Extraction}

According to the Equation (2), the MNDWIs of different Landsat images were calculated to produce water-bodyenhanced images by using the band math module in ENVI software. Then, the MNDWI pixels were stretched to [0, 255], as shown in Figure 4.

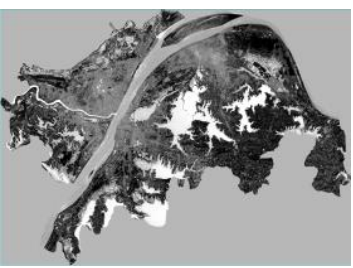

(a)

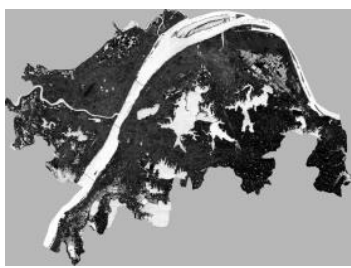

(b) 


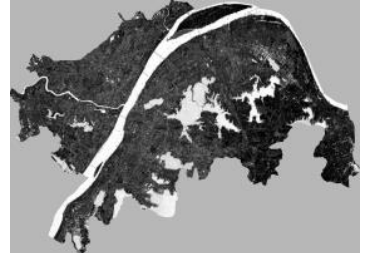

(c)

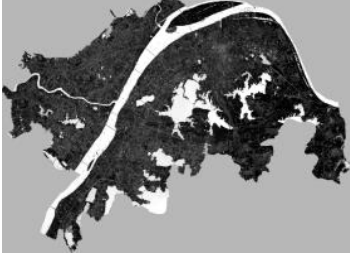

(d)
Figure 4. MNDWI images: (a) 1991, (b) 2002, (c) 2011, and (d) 2017.

In order to identify a suitable threshold for segmentation, histogram statistics of MNDWI images were then performed using ENVI. Taking Figure 4 (c) as an example, the histogram was a bimodal distribution (Figure 5). Since the OTSU algorithm was implemented using MATLAB in this study, the histogram statistic data were exported to MATLAB to calculate optimal threshold. The OTSU algorithm indicated that the best threshold of Figure 4 (c) was 95.6250, the corresponding normalized value was -0.3497 . Spectral analysis of the pixels in the range $[-0.3497,0]$ shown that there is a big difference between the spectral curves of pure water pixel and other pixel, -0.3497 was thus an optimal threshold for separating water from other land cover features.

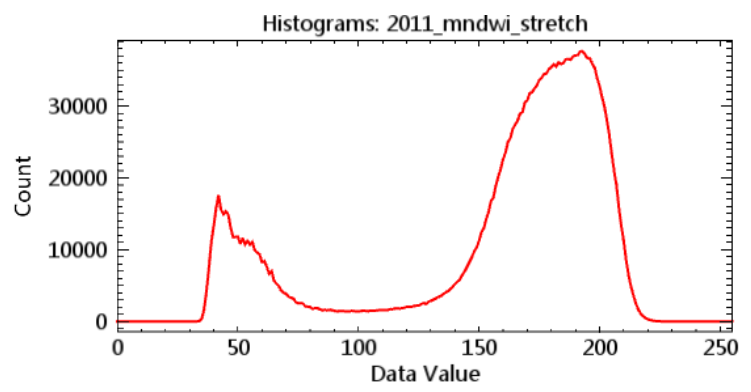

Figure 5. 2011 MNDWI image histogram.

Based on the optimal threshold, a binary image of the water body was obtained by threshold segmentation of each MNDWI image, which were shown in Figure 6.

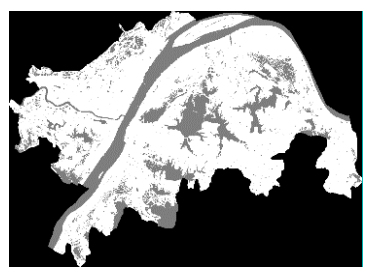

(a)

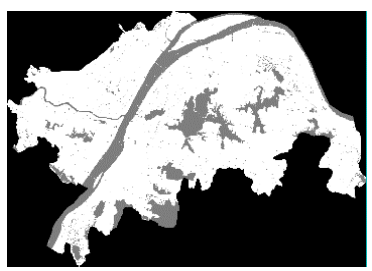

(c)

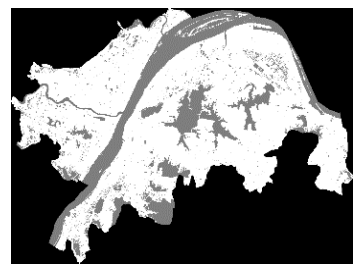

(b)

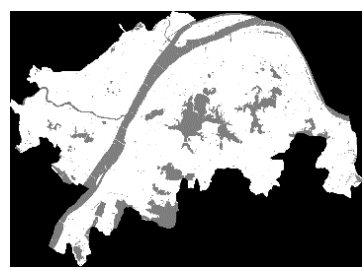

(d)
Figure 6. Binary maps of different MNDWI images based on OTSU segmentation: (a) 1991, (b) 2002, (c) 2011, and (d) 2017.
Considering the extracted water bodies contained the Yangtze River and other small river, an accurate vectorization was carried out to get the shapes of urban lakes (Figure 7).

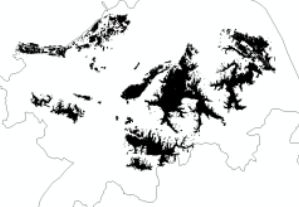

(a)

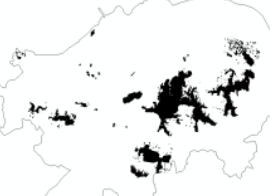

(c)

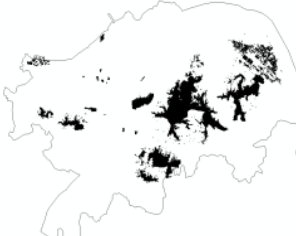

(b)

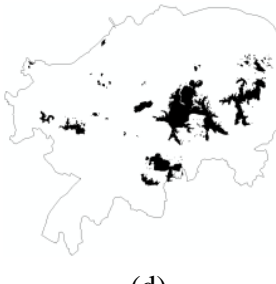

(d)
Figure 7. Vector maps of extracted urban lakes in different year: (a) 1991, (b) 2002, (c) 2011, and (d) 2017.

Then, the area of urban lakes can be easily calculated from these vector maps. The statistics of urban lake areas and the percentage of urban lake area in downtown Wuhan have been presented in Table 2. The results show that the coverage of urban lakes continues to decrease over these years, and the proportion of urban lake areas in the downtown has dropped by $4.93 \%$ from 1991 to 2017 . The total area of urban lakes has been reduced by $47.37 \mathrm{~km}^{2}$ during the past 27 years, which indicates that the urban lakes of Wuhan had experienced significant shrink or disappearance in this period. The most intense decrease of urban lakes was detected between 1991 and 2002, during which the surface area of urban lakes lost 38.97 $\mathrm{km}^{2}$, over $36.07 \%$ of their original surface area was reduced in comparison with the year 1991. From 2002 to 2011, the decrease of urban lakes cannot be ignored as well, there was $6.52 \mathrm{~km}^{2}$ lake area lost. In the last 7 years, the destruction of urban lakes is much smaller than before.

\begin{tabular}{|c|c|c|}
\hline Year & Urban lakes area $\left(\mathrm{km}^{2}\right)$ & Percentage of lake area (\%) \\
\hline 1991 & 108.01 & 11.23 \\
\hline 2002 & 69.04 & 7.17 \\
\hline 2011 & 62.52 & 6.49 \\
\hline 2017 & 60.64 & 6.30 \\
\hline
\end{tabular}

Table 2. Area statistics of Wuhan urban lakes over the years.

\subsection{Image Classification}

In order to get detailed changes and factors for urban lakes shrinking, SVM (Support Vector Machine) classification algorithm was further applied to classify the MNDWI image into three classes: water body, built-up area, and vegetation. The classification results of these images are shown in Figure 8. 


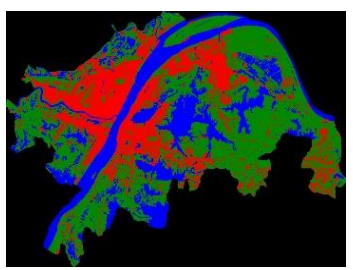

(a)

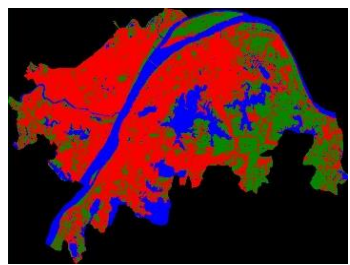

(c)

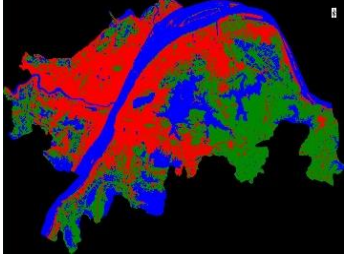

(b)

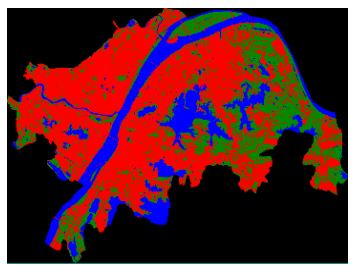

(d)
Water body

Built-up Area

Vegetation

Figure 8 . The classification results of multi-temporal images: (a) 1991, (b) 2002, (c) 2011, and (d) 2017.

The classification accuracy were also evaluated by using confusion matrix. The validation samples for accuracy assessment were carefully selected from each individual imagery by visual interpretation. The classification result of each separate image was quantitatively assessed through the overall accuracy and Kappa coefficients, which was reported in Table 3. It can be seen that an average of $99.16 \%$ overall accuracy was achieved, and the mean Kappa coefficient was 0.9865 .

\begin{tabular}{|l|c|c|}
\hline Image & Overall accuracy (\%) & Kappa coefficient \\
\hline 1991 & 99.77 & 0.9963 \\
\hline 2002 & 99.81 & 0.9968 \\
\hline 2011 & 98.64 & 0.9791 \\
\hline 2017 & 98.42 & 0.9737 \\
\hline
\end{tabular}

Table 3. The classification accuracy of each Landsat image.

\subsection{Change Detection}

In order to investigate what leads to the intense shrink of urban lakes over the past few decades, post-classification comparison between the four classified images was performed to produce a change detection analysis. Different "from-to" change maps were generated by using ArcGIS 10.2.2 software, which were shown in Figure 9. Post-classification comparison showed that the lost lake surface area had been replaced by a large number of built-up area and vegetation from 1991 to 2017. The changes of the land cover categories were summarized in Table 4 .

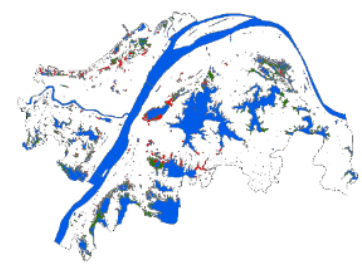

(a)

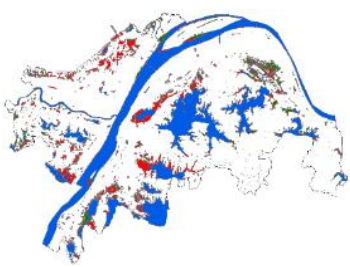

(b)

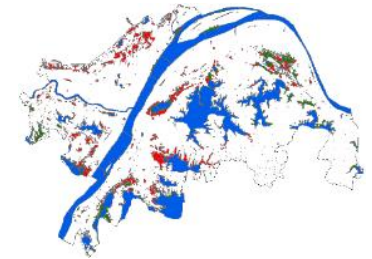

(c)

Wate $\rightarrow$ Built-up Area Water $\rightarrow$ Vegetation $\quad$ Water Figure 9. The post-classification change detection results in different periods: (a) 1991-2002, (b) 1991-2011, and (c) 19912017.

\begin{tabular}{|l|l|l|l|}
\hline Period & Water $\left(\mathrm{km}^{2}\right)$ & $\begin{array}{l}\text { Water to } \\
\text { Vegetation }\left(\mathrm{km}^{2}\right)\end{array}$ & $\begin{array}{l}\text { Water to } \\
\text { Built-up Area } \\
\left(\mathrm{km}^{2}\right)\end{array}$ \\
\hline $1991-2002$ & 193.4397 & 31.9833 & 21.0060 \\
\hline $1991-2011$ & 166.3029 & 23.3226 & 56.8035 \\
\hline $1991-2017$ & 168.1209 & 31.9275 & 46.3806 \\
\hline
\end{tabular}

Table 4. The statistic of change detection results in different periods

From the decrease of urban lakes in the three maps, it can be seen that the central area of Wuhan had mainly experienced intense urbanization. During the period from 1991 to 2002, a large number of lakes were buried or shrink, $52.9893 \mathrm{~km}^{2}$ water were change into built-up area and vegetation during this period. The city was still in a rapid construction stage from 2002 to 2011, urban lakes continued to be devoured by real estate developments and other damages, and the built-up area changed from water bodies had increased by $56.80 \mathrm{~km}^{2}$ between 1991 and 2011. From 2011 to 2017, due to the improvement of local government's lake protection policy, the area of urban lakes did not change much, and the area of vegetation around some lakes began to increase. It indicates that the urbanization process in downtown Wuhan began to slow down. To sum up, the rapid urbanization since 1991 has a significant impact on the losses of urban lakes.

\section{CONCLUSION}

Water extraction and change detection based on remotely sensed images have increasingly been recognized as one of the most effective approaches for environment monitoring and management. This study showed the advantages of using multitemporal Landsat images to perform historical change analysis of urban lakes in respect to specific areas of interest. The experiment results revealed that there were significant changes in the surface area of urban lakes from 1991 to 2017. A large number of urban lakes have shrunk or disappeared during this period, and been replaced by a large number of buildings. The remarkable decrease of urban lakes is witnessed in the expansion of built-up areas. Since the legislation for the protection of urban water resources was not perfect before 2002, the local governments have taken stringent measures to strength the protection and management of urban lakes, such as "The Lake Protection Regulations of Wuhan", and a lake protection plan of "three lines and one way" was also published on December 2012.

In terms of the techniques used in the study, it was found that modified NDWI and OTSU algorithm were able to provide 
rapid and accurate extraction of water information from Landsat multi-spectral images. In addition, post-classification change detection based on SVM algorithm produced high accurate results, which was benefit to detailed land cover change analysis. It can be stated that continuous change detection based on remote sensing techniques enable us to better understand the detailed spatial changes of urban lakes on one hand, and the trends of urban growth on the other hand.

\section{REFERENCES}

Bagan, Hasi, and Yoshiki Yamagata, 2012. Landsat Analysis of Urban Growth: How Tokyo Became the World's Largest Megacity during the Last 40 years. Remote Sensing of Environment, $127, \quad$ pp. 210-222. https://doi.org/10.1016/j.rse.2012.09.011.

Demir, Begüm, Francesca Bovolo, and Lorenzo Bruzzone, 2013. Updating Land-Cover Maps by Classification of Image Time Series: A Novel Change-Detection-Driven Transfer Learning Approach. IEEE Transactions on Geoscience and Remote Sensing, $51 \quad$ (1), 1 pp. 300-312. https://doi.org/10.1109/TGRS.2012.2195727.

Dronova, Iryna, Peng Gong, and Lin Wang, 2011. Object-Based Analysis and Change Detection of Major Wetland Cover Types and Their Classification Uncertainty during the Low Water Period at Poyang Lake, China. Remote Sensing of Environment, 115 (12), pp. 3220-3336. https://doi.org/10.1016/j.rse.2011.07.006.

Ji, Lei, Li Zhang, and Bruce Wylie, 2009. Analysis of Dynamic Thresholds for the Normalized Difference Water Index. Photogrammetric Engineering \& Remote Sensing, 75 (11), pp. 1307-1317. https://doi.org/10.14358/PERS.75.11.1307.

Li, Wenbo, Zhiqiang Du, Feng Ling, Dongbo Zhou, Hailei Wang, Yuanmiao Gui, Bingyu Sun, and Xiaoming Zhang, 2013. A Comparison of Land Surface Water Mapping Using the Normalized Difference Water Index from TM, ETM+ and ALI. Remote Sensing, 5 (11), pp. 5530-5549. https://doi.org/10.3390/rs5115530.

McFeeters, S. K., 1996. The Use of the Normalized Difference Water Index (NDWI) in the Delineation of Open Water Features. International Journal of Remote Sensing, 17 (7), pp. 1425-1432. https://doi.org/10.1080/01431169608948714.

Michishita, Ryo, Zhiben Jiang, and Bing Xu, 2012. Monitoring Two Decades of Urbanization in the Poyang Lake Area, China through Spectral Unmixing. Remote Sensing of Environment, 117, pp. 3-18. https://doi.org/10.1016/j.rse.2011.06.021.

Otsu, Nobuyuki, 1979. A Threshold Selection Method from Gray Level Histogram. IEEE Transactions on Systems Man \& Cybernetics, 9 (1), pp. 62-66.

Rokni, Komeil, Anuar Ahmad, Ali Selamat, and Sharifeh Hazini, 2014. Water Feature Extraction and Change Detection Using Multitemporal Landsat Imagery. Remote Sensing, 6 (5), pp. 4173-4189. https://doi.org/10.3390/rs6054173.

Snehal, Patil, and Padalia Unnati, 2012. Challenges Faced and Solutions towards Conservation of Ecology Of Urban Lakes.
International Journal of Scientific \& Engineering Research, 3 (10), pp. 1-14.

Song, Chunqiao, Bo Huang, and Linghong Ke, 2013. Modeling and Analysis of Lake Water Storage Changes on the Tibetan Plateau Using Multi-Mission Satellite Data. Remote Sensing of Environment, $135, \quad$ pp. 25-35. https://doi.org/10.1016/j.rse.2013.03.013.

Taravat, Alireza, Masih Rajaei, Iraj Emadodin, Hamidreza Hasheminejad, Rahman Mousavian, and Ehsan Biniyaz, 2016. A Spaceborne Multisensory, Multitemporal Approach to Monitor Water Level and Storage Variations of Lakes. Water, 8 (11), pp. 478-486. https://doi.org/10.3390/w8110478.

Wang, Zhaolei, 2013. Study on Protection and Utilization of Wuhan City Lake since 1990s. Environmental Science and Management, 38, pp. 38-45.

Woodcock, Curtis, et al., 2008. Free Access to Landsat Imagery. Science, $320 \quad$ (5879), pp. 1011-1012. https://doi.org/10.1126/science.320.5879.1011a.

$\mathrm{Xu}$, Hanqiu, 2006. Modification of Normalised Difference Water Index (NDWI) to Enhance Open Water Features in Remotely Sensed Imagery. International Journal of Remote Sensing, 27 (14), pp. 3025-3033. https://doi.org/10.1080/01431160600589179.

Zheng, Zhubin, Yunmei Li, Yulong Guo, Yifan Xu, Ge Liu, and Chenggong Du, 2015. Landsat-based Long-Term Monitoring of Total Suspended Matter Concentration Pattern Change in the Wet Season for Dongting Lake, China. Remote Sensing, 7 (10), pp. 13975-13999. https://doi.org/10.3390/rs71013975.

Zhu, Jianfeng, Qiuwen Zhang, and Zhong Tong, 2015. Impact Analysis of Lakefront Land Use Changes on Lake Area in Wuhan, China. Water, 7 (9), pp. 4869-4886. https://doi.org/10.3390/w7094869.

Zhu, Wenbin, Shaofeng Jia, and Aifeng Lv, 2014. Monitoring the Fluctuation of Lake Qinghai Using Multi-Source Remote Sensing Data. Remote Sensing, 6 (11), pp. 10457-10482. https://doi.org/10.3390/rs61110457.

Zhu, Zhe, and Curtis E. Woodcock, 2014. Continuous Change Detection and Classification of Land Cover Using All Available Landsat Data. Remote Sensing of Environment, 144, pp. 152171. https://doi.org/10.1016/j.rse.2014.01.011. 